\title{
Polymorphisms within Genes Coding for IL-17A and F and Their Receptor as Clinical Hallmarks in Ankylosing Spondylitis
}

\author{
Joanna Wielińnska $\mathbb{D}^{1},{ }^{1}$ Jerzy Świerkot $\mathbb{D}^{2},{ }^{2}$ Katarzyna Kolossa, ${ }^{3}$ Bartosz Bugaj $\mathbb{D}^{2}{ }^{2}$ \\ Monika Chaszczewska-Markowska ${ }^{(D)}{ }^{1}$ Sławomir Jeka $\mathbb{D}^{1,}, 4$ \\ and Katarzyna Bogunia-Kubik (1D) \\ ${ }^{1}$ Laboratory of Clinical Immunogenetics and Pharmacogenetics, Hirszfeld Institute of Immunology and Experimental Therapy, \\ Polish Academy of Sciences, R. Weigla 12, 53-114 Wroclaw, Poland \\ ${ }^{2}$ Department of Rheumatology and Internal Medicine, Wroclaw Medical University, Borowska 213, 50-556 Wroclaw, Poland \\ ${ }^{3}$ Department of Rheumatology and Connective Tissue Diseases, Jan Biziel University Hospital No. 2, Ujejskiego 75, \\ 85-168 Bydgoszcz, Poland \\ ${ }^{4}$ Ludwik Rydygier Collegium Medicum in Bydgoszcz, Nicolaus Copernicus University in Torun, Jagiellońska 15, \\ 85-067 Bydgoszcz, Poland
}

Correspondence should be addressed to Katarzyna Bogunia-Kubik; katarzyna.bogunia-kubik@hirszfeld.pl

Received 2 September 2021; Accepted 21 October 2021; Published 27 October 2021

Academic Editor: Radjesh Bisoendial

Copyright (c) 2021 Joanna Wielińska et al. This is an open access article distributed under the Creative Commons Attribution License, which permits unrestricted use, distribution, and reproduction in any medium, provided the original work is properly cited.

\begin{abstract}
IL-17A and IL-17F together with their coreceptor (IL-17RA/RC) were reported to play a significant role in the pathogenesis of spondyloarthritis. The group of axial spondyloarthritis comprises ankylosing spondylitis (AS), a rheumatic disease characterized by chronic inflammation of the joints in the spine. This study is aimed at investigating $I L-17 A, I L-17 F, I L-17 R A$, and $I L-17 R C$ polymorphisms as potential biomarkers of disease susceptibility, clinical parameters, and anti-TNF treatment outcome in a cohort of Polish ankylosing spondylitis patients. In total, 328 subjects, including 138 AS patients and 190 healthy volunteers, participated in the study. Genotyping of $I L-17 A$ rs2275913 (G/A), IL-17F rs763780 (A/G), IL-17RA rs4819554 (A/G), and $I L-17 R C$ rs708567 (G/A) was performed on real-time PCR instrument using LightSNiP assays. No significant differences were revealed in genotype and allele distribution between patients and controls despite the association of the $I L$ $17 R C$ rs708567 AA homozygosity with the earlier onset of the disease. Moreover, some relationships between $I L-17 F$ rs763780 and IL-17RA rs4819554 polymorphisms with clinical parameters related to the disease activity and anti-TNF treatment outcome were observed. IL- $17 F$ rs $763780 \mathrm{G}$ allele was found to be associated with high disease activity and BASDAI after 6 months and poor response to the treatment while higher VAS values were more common among IL-17RA rs4819554 G variant carriers. In conclusion, the $I L-17 F$ rs763780 polymorphism should be considered as a promising biomarker of disease activity and anti-TNF treatment outcome. The $I L-17 R A$ rs48419554 G allele may serve as a potential marker of disease severity in Polish AS patients.
\end{abstract}

\section{Introduction}

Ankylosing spondylitis (AS) is characterized by visible radiographic changes within the spine or sacroiliac joints. The axial spondyloarthritis (axSpA) group comprises AS, radiographic axial spondyloarthritis, and a nonradiographic
(nr-axSpA) form of the disease [1]. AS patients suffer from inflammatory back pain and morning stiffness. Symptoms can also involve enthesitis and peripheral arthritis manifestations. The disease affects mostly men (ratio men to women is 2 to 1 ), those under thirty years of age, and with a strong genetic association linked to HLA-B27 [2]. Prevalence differs 
between geographical regions and ethnicity, reaching $0.23 \%$ in the general European population [3] and roughly $0.083 \%$ in Polish people [4].

The standard pharmacological treatment against AS involves tumour necrosis factor-alpha (TNF- $\alpha$ ) inhibitor (anti-TNF) dosage after the primary failure of nonsteroidal anti-inflammatory (NSAIDs) administration. The longterm anti-TNF approach has positive effects on patient's functional outcome, lessens disease activity, and reduces radiographic progression [5].

The IL-17 family consists of six cytokines: IL-17A, IL17B, IL-17C, IL-17D, IL-17E (IL-25), and IL-17F. Those proteins transmit signals through defined heterodimeric transmembrane receptors (IL-17R). IL-17A, IL-17F, and IL-17A/F heterodimer act via the IL-17RA/RC receptor complex. IL-17E triggers responses through IL-17RA/RB, and IL-17C induces the IL-17RA/RE heterodimer. For other proteins, the heterotrimeric receptor compound has not been fully elucidated. IL-17A and IL-17F have a high degree of homology, and both are secreted by Th17 cells, $\gamma \delta$ T cells, innate lymphoid cells, cytotoxic $\mathrm{T}$ cells, and natural killer $\mathrm{T}$ (NKT) cells [6].

IL-17 was reported to have a crucial role in the immunopathogenesis of spondyloarthritis [7]. Elevated levels of IL17 in serum have been observed in ankylosing spondylitis patients [8]. Besides, associations between IL-17 level and the Bath Ankylosing Spondylitis Disease Activity Index (BASDAI) $[9,10]$ have been described.

However, our knowledge of $I L-17$ gene polymorphisms in AS is still limited. Thus, based on previous research focused on rheumatoid arthritis [11] and osteoarthritis [12], we hypothesized that $I L-17 A$ rs2275913 and $I L-17$ rs763780 might influence AS susceptibility. The targeted single nucleotide polymorphisms were selected based on the available literature, especially on Caucasians, as well as our preliminary experiment on rheumatoid arthritis patients [13]. We also decided to investigate polymorphisms of IL17 receptors $I L-17 R A$ and $I L-17 R C$. IL-17RA rs4819554 was previously linked with response to etanercept in psoriatic arthritis [14], while $I L-17 R C$ rs708567 was associated with lupus arthritis [15] and was described in Tunisians with rheumatoid arthritis [16]. Moreover, the newly performed study considered the $I L-17 R A$ polymorphism as an AS risk factor [17]. To the best of our knowledge, no investigations have been conducted to assess the association between $I L$ $17 R A$ and $I L-17 R C$ polymorphisms and rheumatic diseases in the Polish population.

This study examines the $I L-17 A$ rs2275913, IL-17F rs763780, IL-17RA rs4819554, and $I L-17 R C$ rs708567 genetic variants as potential biomarkers of disease susceptibility, clinical parameters, and anti-TNF treatment outcome in a cohort of Polish AS patients.

\section{Materials and Methods}

2.1. Patients and Controls. One hundred thirty-eight AS patients and one hundred ninety controls were involved in the study. AS patients were recruited from the Department of Rheumatology and Internal Medicine, Wroclaw Medical
University, Poland, and from the Department of Rheumatology and Connective Tissue Diseases, Jan Biziel University Hospital No. 2 in Bydgoszcz, Poland. All the participants diagnosed with AS were Caucasians over 18 years of age, and $74 \%(102 / 138)$ were male. Included criteria comprise a resistance to treatment with at least two nonsteroidal antirheumatic drugs (NSAIDs), high disease activity before starting biological treatment, initialization of anti-TNF therapy at the time of the research, and complete medical history. Subjects with the coexistence of acute or chronic disorders besides AS, other autoimmune diseases, malignancies, or current infections, during pregnancy and breastfeeding, as well as with insufficient clinical records, and an unwillingness or inability to cooperate were excluded from the study.

AS patients were diagnosed according to the 1984 modified New York Criteria [18].

Data such as gender, age, disease onset, disease duration, body mass index (BMI), presence of HLA-B27, C-reactive protein (CRP) level, pain visual analogue scale (VAS, range: 0-100 mm), and Bath Ankylosing Spondylitis Disease Activity Index (BASDAI, range: 0-10) were collected from patients.

$90.4 \%$ of patients were HLA-B27 positive, and most of them $(76.7 \%)$ had the axial form of AS. Drug administration comprised MTX in $27.5 \%$ of cases, corticosteroids $(20.3 \%)$ of subjects and NSAIDs have been taken by $71.3 \%$ of patients.

Bath Ankylosing Spondylitis Disease Activity Index (BASDAI) was used to calculate disease activity, which was considered to be high $(\mathrm{BASDAI} \geq 4)$, moderate $(3 \leq$ BASDAI $<4$ ), or low (BASDAI $<3$ ). The clinical outcome was assessed after 3 and 6 months of anti-TNF treatment. Significant improvement after therapy was defined as a reduction of BASDAI ( $\triangle \mathrm{BASDAI} \geq 2.0$ ), good outcome as $\triangle \mathrm{BASDAI} \geq 2.0$ and $\mathrm{BASDAI}<3.0$ at the endpoint, moderate response as $\triangle \mathrm{BASDAI} \geq 2.0$ and $\mathrm{BASDAI} \geq 3.0$ at the endpoint, and no improvement as $\triangle \mathrm{BASDAI}<2.0$ [19].

The patient's demographic and clinical characteristics are described in Table 1. The data are presented as median with range (minimum to maximum).

The control group was enrolled from the healthy volunteers, 63 females (33\%) and 127 (67\%) males, from the Regional Centre of Transfusion Medicine and Blood Bank in Wroclaw without a personal history of rheumatic diseases.

Informed consent was obtained from all participants involved in the study. The research was approved by the Wroclaw Medical University Ethics Committee (identification code KB-625/2016).

2.2. SNP Selection and Genotyping. Tested genetic variants were selected based on analysis of previous publications and search results from NCBI Database of Short Genetic Variations (dbSNP) and SNPinfo Web Server [20]. Minor allele frequency (MAF) in EUR was above 5\% (1000 Genomes Project) $[21]$.

In total, four single nucleotide polymorphisms (SNPs) were chosen for analysis: $I L-17 A$ rs2275913 (G/A) and $I L$ $17 F$ rs763780 (A/G) located on chromosome 6, IL-17RA rs4819554 (A/G) located on chromosome 22, and IL-17RC rs708567 (G/A) located on chromosome 3. Two of them, 
TABle 1: Clinical characteristics of the study cohort.

\begin{tabular}{lcc}
\hline Characteristic & $N$ & Median (range) \\
\hline Age (years) & 138 & $43.5(22-75)$ \\
Disease duration (years) & 135 & $10(0-48)$ \\
Disease onset (years) & 135 & $33(6-56)$ \\
BMI & 113 & $25.32(18.61-40.31)$ \\
CRP before treatment (mg/l) & 108 & $16.83(0.3-561)$ \\
CRP at 3 months (mg/l) & 79 & $5.75(0.2-175)^{*}$ \\
CRP at 6 months (mg/l) & 72 & $5.495(0.2-204.3)^{*}$ \\
VAS before treatment (mm) & 132 & $80(45-100)$ \\
VAS at 3 months (mm) & 138 & $30(0-80)^{*}$ \\
VAS at 6 months (mm) & 131 & $20(0-100)^{*}$ \\
BASDAI before treatment & 138 & $8(4.05-10)$ \\
BASDAI at 3 months & 138 & $3.2(0.7-6.7)^{*}$ \\
BASDAI at 6 months & 132 & $2.25(0.2-9.75)^{*}$ \\
Treatment (anti-TNF drug) & $N=138$ & $n(\%)$ \\
Adalimumab & & $63(45.6 \%)$ \\
Etanercept & & $44(31.9 \%)$ \\
Certolizumab & & $17(12.3 \%)$ \\
Golimumab & & $12(8.69 \%)$ \\
Infliximab & & $2(1.45 \%)$ \\
\hline
\end{tabular}

$N$ : number of patients with clinical information; BMI: body mass index; CRP: C-reactive protein; MTX: methotrexate; NSAIDs: nonsteroidal antiinflammatory drugs; BASDAI: Bath Ankylosing Spondylitis Disease Activity Index; VAS: visual analogue scale; HLA-B27: human leukocyte antigen B27. ${ }^{*} p<0.001 ; p$ value comparing the clinical variables between baseline and after 3 or 6 months of treatment.

$I L-17 F$ rs763780 and $I L-17 R C$ rs708567 are missense variants in exon 3 (His161Arg) and exon 4 (Ser111Leu), respectively. IL-17A rs2275913 and IL-17RA rs4819554 are substitutions within gene promoter regions with a predicted transcription factor binding site (TFBS).

Whole blood samples were collected in EDTA tubes (BD Vacutainer $^{\circledR}$ Blood Collection Tubes). Genomic DNA was isolated from peripheral blood using QIAamp DNA Blood Midi/Maxi Kit (Qiagen, Hilden, Germany) following the manufacturer's protocol. The genotyping of selected SNPs: $I L-17 A$ rs2275913 (G/A), IL-17F rs763780 (A/G), IL-17RA rs4819554 (A/G), and $I L-17 R C$ rs708567 (G/A) was performed using LightSNiP assays (TIB MOLBIOL, Berlin, Germany) on the LightCycler 480 Real-Time PCR Instrument (Roche Diagnostics, Basel, Switzerland).

2.3. Statistical Analysis. The genotype frequencies were tested for the Hardy-Weinberg equilibrium (HWE). Potential differences in allele and genotype distributions between the patient and control groups were calculated using Fisher's exact test. Continuous variables were tested for normal distribution by the Shapiro-Wilk test. Quantitative variables that were normally distributed were presented as mean \pm SEM, while medians with interquartile ranges (IQRs) were calculated for nonnormally distributed variables. The unpaired two-sample Wilcoxon test (for nonparametric data) or unpaired twosample $t$-test (for normally distributed data) were performed
TABLE 2: The distribution of $I L-17$ genotypes and alleles in AS patients and the control group.

\begin{tabular}{|c|c|c|}
\hline & Patients & Controls \\
\hline$I L-17 A$ rs 2275913 & $N=138$ & $N=190$ \\
\hline$G$ & $174(63.0 \%)$ & $234(61.6 \%)$ \\
\hline$A$ & $102(37.0 \%)$ & $146(38.4 \%)$ \\
\hline$G G$ & $50(36.2 \%)$ & $69(36.3 \%)$ \\
\hline$G A$ & $74(53.6 \%)$ & $96(50.5 \%)$ \\
\hline$A A$ & $14(10.1 \%)$ & $25(13.2 \%)$ \\
\hline$I L-17 F$ rs763780 & $N=138$ & $N=189$ \\
\hline$A$ & $265(96.0 \%)$ & $359(95.0 \%)$ \\
\hline$G$ & $11(4.0 \%)$ & $19(5.0 \%)$ \\
\hline$A A$ & $127(92.0 \%)$ & $170(89.9 \%)$ \\
\hline$A G$ & $11(7.97 \%)$ & $19(10.1 \%)$ \\
\hline$G G$ & $0(0 \%)$ & $0(0 \%)$ \\
\hline$I L-17 R A$ rs4819554 & $N=138$ & $N=190$ \\
\hline$A$ & $215(77.9 \%)$ & $311(81.8 \%)$ \\
\hline$G$ & $61(22.1 \%)$ & $69(18.2 \%)$ \\
\hline$A A$ & $83(60.1 \%)$ & $126(66.3 \%)$ \\
\hline$A G$ & 49 (35.5\%) & $59(31.1 \%)$ \\
\hline$G G$ & $6(4.35 \%)$ & $5(2.63 \%)$ \\
\hline$I L-17 R C$ rs708567 & $N=138$ & $N=189$ \\
\hline$A$ & $150(54.3 \%)$ & $205(54.2 \%)$ \\
\hline$G$ & $126(45.7 \%)$ & $173(45.8 \%)$ \\
\hline$A A$ & $41(29.7 \%)$ & $47(24.9 \%)$ \\
\hline$A G$ & $68(49.3 \%)$ & $111(58.7 \%)$ \\
\hline$G G$ & $29(21.0 \%)$ & $31(16.4 \%)$ \\
\hline
\end{tabular}

to identify associations within genetic variants and clinical parameters. Fisher's exact test was also applied to detect relationships between genotypes and categorical variables such as disease activity or treatment outcome. A $p$ value lower than $0.05(p<0.05)$ was considered statistically significant. All statistical analysis was performed using R Software (http://www .r-project.org) and GraphPad Prism 7 for Windows.

\section{Results}

3.1. Distribution of $I L-17 A, I L-17 F, I L-17 R A$, and $I L-17 R C$ Alleles and Genotypes in Patients and Controls. The distribution of genotypes and alleles of $I L-17 A$ rs2275913, IL-17F rs763780, $I L-17 R A$ rs4819554, and $I L-17 R C$ rs708567 did not differ between AS patients and healthy individuals (Table 2). Also, no significant gender-dependent differences were detected between patients and healthy subjects (not shown). Please note that none of the patients or controls were homozygous for the $I L-17 F$ rs763780 G allele. Thus, in the further analyses, $A A$ homozygotes were being compared with $A G$ genotype reflecting also the $G$ allele carriers.

On the other hand, the significant association between disease onset and genotype frequency was observed for $I L$ $17 R C$ rs708567 SNP. Patients with $A A$ genotype had a lower age of disease onset $(29.39 \pm 1.405)$ than those with $G$ allele $(33.43 \pm 1.001)(A A$ vs. $A G+G G, p=0.022$; $A A$ vs. $A G, p=$ 
0.015). However, disease duration was not found to be affected by any of the analysed SNPs.

3.2. Associations between IL-17A, IL-17F, IL-17RA, and IL17RC Genotypes and Clinical Parameters. The potential associations between $I L-17 A, I L-17 F, I L-17 R A$, and $I L$ $17 R C$ genotypes and CRP level, VAS, and BASDAI values were analysed.

During anti-TNF therapy, these major clinical parameters were decreased. CRP level was significantly lower after 3 and 6 months compared to baseline $(p<0.0001)$. Also, VAS and BASDAI were reduced after 3 and 6 months related to baseline, as well as after 6 months in comparison to 3 months of therapy $(p<0.0001)$ (Table 1$)$. A significant improvement in clinical parameters was achieved after administration of anti-TNF agents.

Higher VAS values at the baseline were found in $I L-17 A$ rs2275913 $G G$ ( $G G$ vs. $G A+A A, p=0.005$; $G G$ vs. $G A, p=$ $0.006)$ and $I L-17 F \operatorname{rs} 763780 A G$ ( $A A$ vs. $A G, p=0.027)$ genotype carriers (Table 3(a)).

The $I L-17 R A$ rs4819554 G allele was found to be more common among patients who presented with higher VAS and BASDAI values after anti-TNF treatment induction.

Patients possessing the $I L-17 R A$ rs $4819554 \mathrm{G}$ allele had higher VAS values after 3 months of anti-TNF therapy ( $A A$ vs. $A G+G G, p=0.002$ ). This result was also observed after 6 months of treatment ( $A A$ vs. $A G+G G, p=0.002$ ).

Besides, IL-17RA rs4819554 G patients demonstrated greater BASDAI values at 6 months of therapy than $A A$ homozygotes ( $A A$ vs. $A G+G G, p=0.046$ ) (Table $3(\mathrm{~b})$ ). The $I L-17 R A$ results concerning VAS at 3 and 6 months and BASDAI at 6 months were confirmed by the overdominant model $(A A+G G$ vs. $A G, p=0.008, p=0.006$, and $p=0.045$, respectively). Additionally, a significant relationship with an absolute BASDAI change ( $\triangle$ BASDAI 0-3m.) $(A A+G G$ vs. $A G, p=0.027)$ was noted. Tendencies were observed regarding BASDAI score at 3 months ( $A A$ vs. $A G+G G, p=$ 0.066; AA+GG vs. AG, $p=0.064)$ and absolute BASDAI change ( $\triangle$ BASDAI 0-6 m.) $(A A+G G$ vs. $A G, p=0.057)$.

The BASDAI parameter at 6 months was also higher in the group of AS individuals bearing $I L-17 F$ rs763780 AG genotype ( $G$ allele) ( $A A$ vs. $A G, p=0.035)$ in comparison to $A A$ carriers (Table $3(\mathrm{a}))$.

As for CRP levels, IL-17RC rs708567 and IL-17A rs2275913 polymorphisms were identified as significantly associated with CRP level after 3 months of TNF inhibitor administration. At that time point, $I L-17 A$ rs2275913 GG was more frequently observed among patients with an elevated level of CRP ( $>10 \mathrm{mg} / \mathrm{l})$ ( $G G$ vs. $G A+A A, p=0.022$, $\mathrm{OR}=3$, and $95 \% \mathrm{CI}=1.237-7.046)$, and $I L-17 R C$ rs708567 $\mathrm{G}$ patients showed a significantly higher CRP level as compared to the $A A$ patients ( $A A$ vs. $A G+G G, p=0.043 ; A A$ vs. $A G, p=0.018$ ) (Table $3(\mathrm{~b})$ ).

No other significant differences between clinical parameters of AS patients and their $I L-17$ genotype distribution were detected.

3.3. Effect of IL-17F Polymorphisms on the Disease Activity and Anti-TNF Treatment Outcome. Before the anti-TNF administration, all patients were characterized with high disease activity (BASDAI $>4$ ). After 3 months of therapy, $25.4 \%$ (35/138), 44.9\% (62/138), and $29.7 \%$ (41/138) of subjects had a high, moderate, and low disease activity, respectively. After 6 months, only 3.03\% (4/132) and $1.52 \%$ (2/132) were described with high and moderate disease activity, respectively. The remaining 95.5\% (126/132) of patients presented low disease activity. After 3 months of anti-TNF treatment, $97.8 \%(135 / 138)$ achieved a good or moderate outcome, and $2.2 \%(3 / 128)$ were nonresponders. Similarly, after 6 months, 3\% (4/132) of patients did not respond positively to treatment.

Out of $I L-17 A, I L-17 F, I L-17 R A$, and $I L-17 R C$ polymorphisms studied, a significant association concerning disease activity was detected for the $I L-17 F$ rs763780 variant. AS patients homozygous for the $A$ allele more likely presented low or moderate disease activity $(\mathrm{BASDAI}<4)$ after 6 months of treatment than heterozygotes ( $A A$ vs. $A G, p=$ 0.035 , $\mathrm{OR}=13.22$, and $95 \% \mathrm{CI}=1.82-87.84)$. The same genotype was significantly more common among subjects with a good or moderate response to TNF inhibitor therapy ( $A A$ vs. $A G, p=0.035, \mathrm{OR}=13.22$, and $95 \% \mathrm{CI}=1.82-87.84$ ).

The other studied IL-17A rs2275913, IL-17RA rs4819554, and $I L-17 R C$ rs708567 genetic variants were not found to significantly differ among AS patients in respect to disease activity and biological agent treatment outcome.

\section{Discussion}

In the present study, patients with ankylosing spondylitis and controls were genotyped for the $I L-17 A, I L-17 \mathrm{~F}, I L-$ $17 R A$, and $I L-17 R C$ polymorphisms to assess whether their genetic variants may be associated with susceptibility to the disease, clinical parameters, and anti-TNF treatment outcome in our Polish population.

Comparison made between our patients and controls did not show any significant association with predisposition to the disease as in both groups, similar distributions of alleles and genotypes of all SNPs studied were observed. However, disease onset was found to be affected by the $I L-17 R C$ rs708567 SNP and the presence of $A A$ homozygosity prevailed in patients that had a lower age of disease onset than those with $G$ allele.

Among currently analysed genetic variants, $I L-17 A$ rs2275913 and $I L-17 F$ rs763780 have been previously extensively studied for associations with various rheumatic disorders.

In our current study, neither $I L-17 A$ rs2275913 nor $I L$ $17 F$ rs763780 was found to be associated with AS risk. Nevertheless, association with AS susceptibility and $I L-17 A$ rs2275913 in Chinese [22] and $I L-17 F$ rs763780 in Turkish [23] populations has been reported. Erkol et al. did not find the relationship between $I L-17 A$ rs2275913 and AS susceptibility in Turkish patients [23]. More recently, Rocha Loures et al. reported rs2275913 $A$ variant and rs763780 $\mathrm{G}$ allele as risk factors for AS, spondyloarthritis ( $\mathrm{ppA})$, and psoriatic arthritis (PsA) in Brazilian patients [24].

As for the associations of $I L-17$ polymorphisms with other diseases, many previous studies focused on 


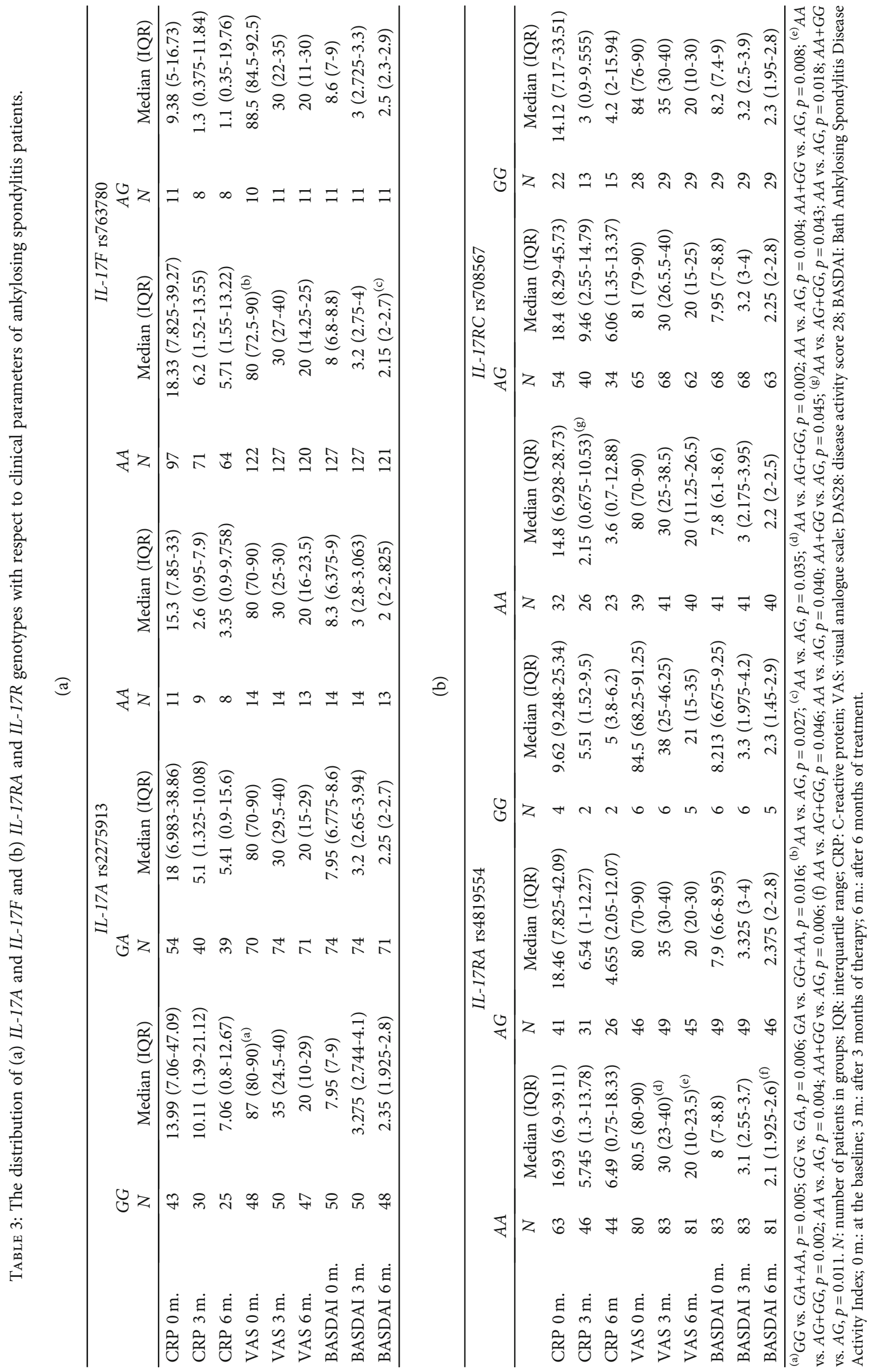


osteoarthritis (OA) and rheumatoid arthritis (RA). Results of analysis performed on an Asian OA group suggested that the $I L-17 A$ rs2275913 $A$ allele [25-27] and $I L-17 F$ rs763780 G variant [26] increased susceptibility to knee OA. In Caucasians, no association between $I L-17 A$ rs2275913 polymorphism and risk of hip or knee OA was found [28], but the $I L$ $17 F$ rs763780 $\mathrm{G}$ allele had a significant impact on the risk of the hip [28] and knee OA [29]. IL-17A rs2275913 GA [29], $I L-17 F$ rs763780 AA [28], and IL-17A-F G-A haplotype [30] seem to play a rather protective role in the knee, hip, or hip and knee OA, respectively. Further meta-analysis performed by Lu et al. highlighted higher susceptibility to OA in patients with $I L-17 A$ rs $2275913 A$ allele and $I L-17 F$ rs 763780 $\mathrm{G}$ allele among Asians, as well as with $I L-17 F$ rs763780 G genetic variant in a Caucasian cohort [12].

Interestingly, papers concerning the role of $I L-17 \mathrm{~A}$ rs2275913 and $I L-17 F$ rs763780 in RA are inconsistent. $I L$ $17 A$ rs $2275913 G G$ genotype $[31,32]$ and $G$ allele [33] have been found to increase susceptibility to RA, whereas Shen et al. described $A A$ genotype as being linked to lower RA risk [34]. Other studies showed no significant correlations between IL-17A rs2275913 variant and prevalence to develop RA in Polish [13, 35], Turkish [36], Brazilian [37], Tunisian [16, 38], Algerian [39], Mexican [40], and Egyptian [41] patients. Growing evidence suggests that $I L-17 F$ rs763780 $\mathrm{G}$ is associated with susceptibility to the disease $[13,33,38]$. However, many studies did not confirm this polymorphism as an RA risk factor [35-37, 39, 41, 42]. Recent meta-analysis findings led to the identification of $I L-17 A G G$ and $I L-17 F A G$ genotypes as more frequently distributed among RA patients [11].

With regard to $I L-17 R A$ and $I L-17 R C$ polymorphisms, the present analysis did not show differences in genotype and allele distribution between patients and controls. This observation confirms previous results for IL-17RC among RA patients [16] but stays in contrast with $I L-17 R A$ developments in AS Spanish cohorts [17]. IL-17RA rs48419554 was also identified as a risk factor for psoriasis $[43,44]$. However, other IL-17RA SNPs were not found to be associated with PsA [45].

Our current results also show that $I L 7 R C$ rs708567 G variant has an effect on disease onset and is more frequently detected among patients that developed the diseases approximately 4 years later than $A A$ homozygotes. To the best of our knowledge, no one has studied $I L-17 R C$ rs708567 in AS so far. This genetic variant and its homozygosity were also described to affect arthritis among systemic lupus erythematosus Bulgarian patients [46]. On the other hand, in a study conducted by Dhaouadi et al., IL7RC A allele of this polymorphism tended to show higher DAS28 in RA subjects [16]. However, the functional consequence of $I L-17 R C$ rs708567 polymorphism remains unknown.

In the present study, some interesting results were described regarding the $I L-17 F$ rs763780 SNP and our cohort of patients with AS and unfavourable effect of the IL-17F rs763780 G allele.

Likewise, the $I L-17 F$ rs763780 $G$ allele was observed by Paradowska-Gorycka et al. to be positively correlated with the number of tender joints, as well as to tend to reach insig- nificantly higher values of DAS28-CRP and health assessment questionnaire (HAQ) score [42].

According to earlier findings established in Turkey, the $I L-17 F$ rs763780 GG genotype was prone to greater BASFI scores and $A G$ variant to higher CRP level [23]. As suggested, the evidence we found points to an association between this polymorphism and disease activity in AS patients. Our results show that the $A G$ genotype is significantly correlated with higher, both VAS values before treatment and BASDAI score after 6 months. Also, we link $A A$ genotype with moderate/low disease activity and good/moderate response to treatment after 6 months. This concurs well with results obtained by Prieto-Peréz et al., who observed that rs763780 can predict response to adalimumab at 6 months, in psoriasis [47].

Of note, our previous analysis showed the association of the $I L-17 F$ rs763780 G allele with higher IL-17F secretion [48]. Also, Braga et al. observed this association between the $I L-17 F$ rs763780 G allele and increased IL-17F serum levels in Brazilian AS patients and controls [49]. These results suggest that alleles or genotypes associated with higher IL-17F production may play an unfavourable role.

Recently, the novel insight into functional consequences of the IL-17F polymorphism was described by Nisar et al. The change at position 161 (His to Arg) is located in the C-terminal end of IL-17, which interacts with IL-17RA. This substitution resulted in more favourable conformation, enhanced stability of the trimeric IL-17A/F/IL-17RA complex. The stronger binding may induce the proinflammatory effect and influence the severity of RA [50].

One of our previous studies performed on RA patients found that $I L-17 A$ rs $2275913 G G$ homozygous females were characterized with the most active disease after 3 months and poor response to anti-TNF therapy [13]. On the other hand, de la Peña et al. reported that $A$ allele carriers were predicted to present more severe RA and needed more than three DMARDs to control the disease [51].

It has been reported that the IL-17A rs2275913 polymorphism located in the promoter region can regulate gene transcription and stimulate IL-17 cytokine secretion (-197A allele) [52].

Current analysis demonstrates a significant correlation between the IL-17A GG genotype and higher VAS values before starting therapy in AS patients. The same genotype more frequently characterized patients with elevated CRP after 3 months. We did not find any significant correlation between $I L-17 \mathrm{~A}$ variants and response to the therapy. However, it was reported in the literature that rs2275913 was associated with response to anti-TNF therapy among patients with inflammatory bowel disease [53].

Our results are also in line with the findings of VidalCastiñeira et al. concerning significantly higher BASDAI scores in AS patients carrying the IL-17RA rs $4819554 \mathrm{G}$ allele. Moreover, our study reveals associations between the $I L-17 \mathrm{~A}$ $G$ allele and greater VAS after 3 and 6 months of anti-TNF treatment. These correlations are worth mentioning because they indicate an impact of $G$ variant, located in the gene promoter, on AS severity. This region was also noted to affect the response to anti-TNF therapy outcome in psoriasis [54]. 
The IL-17RA rs4819554 variant is encoded within the promotor region and may have a functional effect by modulating the gene transcription. This SNP was found to be in linkage disequilibrium with rs4819553 and rs4819958. Those polymorphisms are predicted to be related to transcription factor binding sites (TFBSs) belonging to the Ikaros (IK) family. These are involved in Th17 cell differentiation [54].

In $G$ allele carriers, the increase of Th17 cytokines could promote the pathogenic mechanism via IL-23/Th17 pathway [54]. It could explain the higher VAS values and BASDAI scores that we observed.

These findings shed some light on common genetic variants in $I L-17 A, I L-17 F$, and $I L-17 R A$ genes. The investigated polymorphisms can affect biological activity of the protein and thus influence immunological features like a response to etanercept [14].

There is still considerable controversy surrounding $I L-17$ SNP relationships and AS development. Therefore, our results need to be interpreted with caution. In fact, population diversity and treatment approach may explain the differences between studies. Although the advantage of our methodology is homogeneity of the Polish population, we are aware that the main limitation of our study is the relatively limited number of cases included in the analysis. Therefore, further data collection from AS patients is required to confirm these observations.

Other interesting genetic variants within $I L-17 \mathrm{~F}$ include rs11465553 [35] and rs2397084 [33, 35, 36, 38, 39, 42], which were investigated in RA. In a Polish cohort, the rs2397084 polymorphism was correlated with longer disease duration [42], whereas in Tunisians, it was associated with disease severity [38]. Additionally, rs2397084 [29] and rs $1889570[28,30]$ were studied in osteoarthritis patients. Regarding the $I L-17 \mathrm{~A}$ gene, rs3804513 was associated with radiographic progression in early RA [55]. Other IL-17A polymorphisms were studied in a Chinese population. $I L$ $17 A$ rs4711998 and rs8193037 were not associated with RA, whereas rs3819024, rs3819025, and rs8193036 were correlated with the risk of RA [34]. These polymorphisms may be of interest for further study on ankylosing spondylitis.

\section{Conclusions}

The analysis shows that $I L-17$ polymorphisms are associated with clinical parameters in Polish patients with ankylosing spondylitis and have influence on AS severity and potential course of the disease and may be biomarkers of response to anti-TNF drugs in Polish patients. The IL-17F rs763780 polymorphism should be considered as a candidate biomarker of disease activity and anti-TNF treatment outcome. The IL-17RA rs48419554 G allele may serve as a potential marker of disease severity.

\section{Data Availability}

The data that support the findings of this study are available from the corresponding author upon reasonable request.

\section{Conflicts of Interest}

The authors declare that there is no conflict of interest.

\section{Authors' Contributions}

Conceptualization was contributed by K.B.-K. and J.W.; methodology was done by J.W. and K.B.-K.; formal analysis was carried out by J.W. and K.B.-K.; investigation was done by J.W. and K.B.-K.; resources were contributed by J.S., K.K., B.B., M.Ch.-M., S.J., and K.B.-K.; data curation was performed by J.W., J.S., K.K., B.B., M.Ch.-M., and S.J.; writing-original draft preparation was contributed by J.W. and K.B.-K.; writing-review and editing was contributed by J.S., K.K., B.B., M.Ch.-M., S.J., and K.B.-K.; supervision was contributed by J.S., S.J., and K.B.-K.; project administration was done by K.B.-K.; funding acquisition was contributed by K.B.-K. All authors have read and agreed to the published version of the manuscript.

\section{Acknowledgments}

This research was funded by the National Science Centre (Poland) (grant number 2016/21/B/NZ5/01901). The authors thank the Regional Centre of Transfusion Medicine and Blood Bank in Wroclaw for providing control samples.

\section{References}

[1] S. P. Raychaudhuri and A. Deodhar, "The classification and diagnostic criteria of ankylosing spondylitis," Journal of Autoimmunity, vol. 48-49, pp. 128-133, 2014.

[2] J. Sieper and D. Poddubnyy, "Axial spondyloarthritis," in The Lancet, pp. 73-84, Lancet Publishing Group, 2017.

[3] L. E. Dean, G. T. Jones, A. G. Macdonald, C. Downham, R. D. Sturrock, and G. J. Macfarlane, "Global prevalence of ankylosing spondylitis," Rheumatol (United Kingdom), vol. 53, pp. 650-657, 2014.

[4] M. Tłustochowicz, M. Brzozowska, W. Wierzba et al., "Prevalence of axial spondyloarthritis in Poland," Rheumatology International, vol. 40, pp. 323-330, 2020.

[5] M. S. Osman and W. P. Maksymowych, "An update on the use of tumor necrosis factor alpha inhibitors in the treatment of ankylosing spondylitis," Expert Review of Clinical Immunology, vol. 13, no. 2, pp. 125-131, 2017.

[6] L. Monin and S. L. Gaffen, "Interleukin 17 family cytokines: signaling mechanisms, biological activities, and therapeutic implications," Cold Spring Harbor Perspectives in Biology, vol. 10, 2018.

[7] H. Tsukazaki and T. Kaito, "The role of the IL-23/IL-17 pathway in the pathogenesis of spondyloarthritis," International Journal of Molecular Sciences, vol. 21, pp. 1-19, 2020.

[8] Y. Mei, F. Pan, J. Gao et al., "Increased serum IL-17 and IL-23 in the patient with ankylosing spondylitis," Clinical Rheumatology, vol. 30, pp. 269-273, 2011.

[9] W.-S. Chen, Y.-S. Chang, K.-C. Lin et al., "Association of serum interleukin-17 and interleukin-23 levels with disease activity in Chinese patients with ankylosing spondylitis," Journal of the Chinese Medical Association, vol. 75, pp. 303-308, 2012 . 
[10] L. Xueyi, C. Lina, W. Zhenbiao, H. Qing, L. Qiang, and P. Zhu, "Levels of circulating Th17 cells and regulatory T cells in ankylosing spondylitis patients with an inadequate response to antiTNF- $\alpha$ therapy," Journal of Clinical Immunology, vol. 33, pp. 151-161, 2013.

[11] I. Agonia, J. Couras, A. Cunha, A. J. Andrade, J. Macedo, and B. Sousa-Pinto, "IL-17, IL-21 and IL-22 polymorphisms in rheumatoid arthritis: A systematic review and _meta_ -analysis," Cytokine, vol. 125, p. 154813, 2020.

[12] F. Lu, P. Liu, Q. Zhang, W. Wang, and W. Guo, "Association between the polymorphism of IL-17A and IL-17F gene with knee osteoarthritis risk: a meta-analysis based on casecontrol studies," Journal of Orthopaedic Surgery and Research, vol. 14, 2019.

[13] K. Bogunia-Kubik, J. Świerkot, A. Malak et al., "IL-17A, IL-17F and IL-23R gene polymorphisms in polish patients with rheumatoid arthritis," Archivum Immunologiae et Therapiae Experimentalis (Warsz), vol. 63, pp. 215-221, 2015.

[14] G. Murdaca, S. Negrini, O. Magnani, E. Penza, M. Pellecchio, and F. Puppo, "Impact of pharmacogenomics upon the therapeutic response to etanercept in psoriasis and psoriatic arthritis," Expert Opinion on Drug Safety, vol. 16, pp. 1173-1179, 2017.

[15] M. Hristova, Z. Kamenarska, G. Dzhebir et al., "The role of IL17 rs2275913, IL-17RC rs708567 and TGFB1 rs1800469 SNPs and IL-17A serum levels in patients with lupus nephritis," Rheumatology International, 2021.

[16] T. Dhaouadi, M. Chahbi, Y. Haouami et al., "IL-17A, IL-17RC polymorphisms and IL17 plasma levels in Tunisian patients with rheumatoid arthritis," PLoS One, vol. 13, 2018.

[17] J. R. Vidal-Castiñeira, A. López-Vázquez, R. Diaz-Peña et al., "A single nucleotide polymorphism in the Il17ra promoter is associated with functional severity of ankylosing spondylitis," PLoS One, vol. 11, 2016.

[18] S. van der Linden, H. A. Valkenburg, and A. Cats, "Evaluation of diagnostic criteria for ankylosing spondylitis. A proposal for modification of the New York criteria," Arthritis and Rheumatism, vol. 27, pp. 361-368, 1984.

[19] J. Zochling and J. Braun, "Assessment of ankylosing spondylitis," Clinical and Experimental Rheumatology, vol. 23, pp. S133-S141, 2005.

[20] Z. Xu and J. A. Taylor, "SNPinfo: integrating GWAS and candidate gene information into functional SNP selection for genetic association studies," Nucleic Acids Research, vol. 37, pp. W600-W605, 2009.

[21] A. Auton, G. R. Abecasis, D. M. Altshuler, R. M. Durbin, D. R. Bentley, and A. Chakravarti, "A global reference for human genetic variation,” Nature, vol. 526, no. 7571, pp. 68-74.

[22] B. Yang, Y. Xu, X. Liu, Z. Huang, and L. Wang, "IL-23R and IL-17A polymorphisms correlate with susceptibility of ankylosing spondylitis in a Southwest Chinese population," Oncotarget, vol. 8, no. 41, pp. 70310-70316, 2017.

[23] E. Erkol Inal, O. Görükmez, S. Eroğlu, Ö. Sağ, and G. Solak, "Associations between polymorphisms of IL-17F and IL17A genes with disease activity and clinical outcome of ankylosing spondylitis," Acta Reumatológica Portuguesa, vol. 2016, 2016.

[24] M. A. Rocha Loures, L. C. Macedo, D. M. Reis et al., "Influence of TNF and IL17 gene polymorphisms on the spondyloarthritis immunopathogenesis, regardless of HLA-B27, in a Brazilian population," Mediators of Inflammation, vol. 2018, 2018.
[25] L. Han, H. S. Lee, J. H. Yoon et al., “Association of IL-17A and IL-17F single nucleotide polymorphisms with susceptibility to osteoarthritis in a Korean population," Gene, vol. 533, pp. 119122,2014

[26] Y. Bai, S. Gao, Y. Liu, S. Jin, H. Zhang, and K. Su, "Correlation between Interleukin-17 gene polymorphism and osteoarthritis susceptibility in Han Chinese population," BMC Medical Genetics, vol. 20, 2019.

[27] L. Jiang, X. Zhou, Y. Xiong, J. Bao, K. Xu, and L. Wu, “Association between interleukin-17A/F single nucleotide polymorphisms and susceptibility to osteoarthritis in a Chinese population," Medicine, vol. 98, 2019.

[28] G. Vrgoc, J. Vrbanec, R. K. Eftedal et al., "Interleukin-17 and Toll-like receptor 10 genetic polymorphisms and susceptibility to large joint osteoarthritis," Journal of Orthopaedic Research, vol. 36, pp. 1684-1693, 2018.

[29] H. H. Bafrani, M. Ahmadi, D. Jahantigh, and M. Karimian, "Association analysis of the common varieties of IL17A and IL17F genes with the risk of knee osteoarthritis," Journal of Cellular Biochemistry, vol. 120, pp. 18020-18030, 2019.

[30] R. K. Eftedal, G. Vrgoc, Z. Jotanovic, and Z. Dembic, “Alternative interleukin $17 \mathrm{~A} / \mathrm{F}$ locus haplotypes are associated with increased risk to hip and knee osteoarthritis," Journal of Orthopaedic Research, vol. 37, pp. 1972-1978, 2019.

[31] I. I. F. G. da Silva, H. D. Angelo, E. Rushansky, M. H. Mariano, M. de Mascena Diniz Maia, and P. R. E. de Souza, "Interleukin (IL)-23 Receptor, IL-17A and IL-17F gene polymorphisms in Brazilian patients with rheumatoid arthritis," Archivum Immunologiae et Therapiae Experimentalis, vol. 65, pp. 537-543, 2017.

[32] G. B. N. Nordang, M. K. Viken, J. E. Hollis-Moffatt et al., "Association analysis of the interleukin 17A gene in Caucasian rheumatoid arthritis patients from Norway and New Zealand," Rheumatology, vol. 48, pp. 367-370, 2009.

[33] A. Amin, N. Sheikh, M. Mukhtar et al., "Association of interleukin-17 gene polymorphisms with the onset of rheumatoid arthritis," Immunobiology, vol. 226, p. 152045, 2021.

[34] L. Shen, H. Zhang, T. Yan, G. Zhou, and R. Liu, “Association between interleukin 17A polymorphisms and susceptibility to rheumatoid arthritis in a Chinese population," Gene, vol. 566, pp. 18-22, 2015.

[35] A. Pawlik, D. Kotrych, D. Malinowski, V. Dziedziejko, M. Czerewaty, and K. Safranow, "IL17A and IL17F gene polymorphisms in patients with rheumatoid arthritis," BMC Musculoskeletal Disorders, vol. 17, pp. 1-6, 2016.

[36] E. E. İnal, O. Görükmez, Ü. Dündar et al., "The influence of polymorphisms of interleukin-17A and -17F genes on susceptibility and activity of rheumatoid arthritis," Genetic Testing and Molecular Biomarkers, vol. 19, pp. 461-464, 2015.

[37] C. N. Carvalho, R. F. do Carmo, A. L. P. Duarte, A. A. T. Carvalho, J. C. Leão, and L. A. Gueiros, "IL-17A and IL-17F polymorphisms in rheumatoid arthritis and Sjögren's syndrome," Clinical Oral Investigations, vol. 20, pp. 495-502, 2016.

[38] O. S. Marwa, T. Kalthoum, K. Wajih, and H. Kamel, “Association of IL17A and IL17F genes with rheumatoid arthritis disease and the impact of genetic polymorphisms on response to treatment," Immunology Letters, vol. 183, pp. 24-36, 2017.

[39] S. Louahchi, I. Allam, L. Berkani, A. Boucharef, A. Abdesemed, and N. Khaldoun, "Association study of single nucleotide polymorphisms of IL23R and IL17 in rheumatoid arthritis in the Algerian population," Acta Reum Port., vol. 41, pp. 151157,2016 
[40] I. Montúfar-Robles, R. E. Barbosa-Cobos, I. Alemán-Ávila, and J. Ramírez-Bello, "IL-17A haplotype confers susceptibility to systemic lupus erythematosus but not to rheumatoid arthritis in Mexican patients," International Journal of Rheumatic Diseases, vol. 22, pp. 473-479, 2019.

[41] F. M. Elfasakhany, M. A. Eldamarawi, and A. E. Khalil, “Association between interleukin-17 gene polymorphism and rheumatoid arthritis among Egyptians," Meta Gene., vol. 16, pp. 226-229, 2018.

[42] A. Paradowska-Gorycka, E. Wojtecka-Lukasik, J. Trefler, B. Wojciechowska, J. K. Lacki, and S. Maslinski, “Association between IL-17F gene polymorphisms and susceptibility to and severity of rheumatoid arthritis (RA)," Scandinavian Journal of Immunology, vol. 72, pp. 134-141, 2010.

[43] D. Sabry, N. Aboraia, and M. Samir, "A potential association between psoriasin to rs4819554 of IL-17RA gene polymorphism in psoriasis Egyptian patients," Archives of Dermatological Research, vol. 312, pp. 273-281, 2020.

[44] A. Batalla, E. Coto, L. González-Lara et al., "Association between single nucleotide polymorphisms IL17RA rs4819554 and IL17E rs79877597 and psoriasis in a Spanish cohort," Journal of Dermatological Science, vol. 80, pp. 111-115, 2015.

[45] M. G. Catanoso, L. Boiardi, P. Macchioni et al., "IL-23A, IL23R, IL-17A and IL-17R polymorphisms in different psoriatic arthritis clinical manifestations in the northern Italian population," Rheumatology International, vol. 33, pp. 1165-1176, 2013.

[46] Z. Kamenarska, M. Hristova, and G. Dzhebir, "Association of IL-17RC rs708567 with systemic lupus erythematosus," Madridge Journal of Dermatology \& Research, vol. 65, pp. 65-67, 2018.

[47] R. Prieto-Pérez, G. Solano-López, T. Cabaleiro et al., “The polymorphism rs763780 in the IL-17F gene is associated with response to biological drugs in patients with psoriasis," Pharmacogenomics, vol. 16, pp. 1723-1731, 2015.

[48] M. Kamińska, W. Niepiekło-Miniewska, A. Nieszporek, K. Gębura, and K. Bogunia-Kubik, "Poster Presentations," HLA, vol. 91, no. 5, pp. 354-474, 2018.

[49] M. Braga, F. F. Lara-Armi, J. S. F. Neves et al., "Influence of IL10 (rs1800896) polymorphism and TNF- $\alpha$, IL-10, IL-17A, and IL-17F serum levels in ankylosing spondylitis," Frontiers in Immunology, vol. 12, 2021.

[50] H. Nisar, U. Pasha, M. U. Mirza et al., "Impact of IL-17F 7488T/C functional polymorphism on progressive rheumatoid arthritis: novel insight from the molecular dynamic simulations," Immunological Investigations, vol. 50, pp. 416-426, 2021.

[51] M. G. de la Peña, R. M. Cruz, E. G. Guerrero, A. G. López, G. P. Molina, and N. E. H. González, "Polymorphism rs2275913 of Interleukin-17A is related to more intensive therapy with disease-modifying anti rheumatic drugs in Mexican patients with rheumatoid arthritis," Acta Reum Port, vol. 42, pp. 155$161,2017$.

[52] J. L. Espinoza, A. Takami, K. Nakata et al., “A genetic variant in the IL-17 promoter is functionally associated with acute graftversus-host disease after unrelated bone marrow transplantation," PLoS One, vol. 6, 2011.

[53] S Bank, P. S. Andersen, J. Burisch et al., "Associations between functional polymorphisms in the NF $\kappa$ B signaling pathway and response to anti-TNF treatment in Danish patients with inflammatory bowel disease," The Pharmacogenomics Journal, vol. 14, pp. 526-534, 2014.
[54] A. Batalla, E. Coto, J. Gómez et al., "IL17RA gene variants and anti-TNF response among psoriasis patients," The Pharmacogenomics Journal, vol. 18, pp. 76-80, 2018.

[55] T. Furuya, M. Hakoda, N. Ichikawa et al., "Associations between HLA-DRB1, RANK, RANKL, OPG, and IL-17 genotypes and disease severity phenotypes in Japanese patients with early rheumatoid arthritis," Clinical Rheumatology, vol. 26, pp. 2137-2141, 2007. 